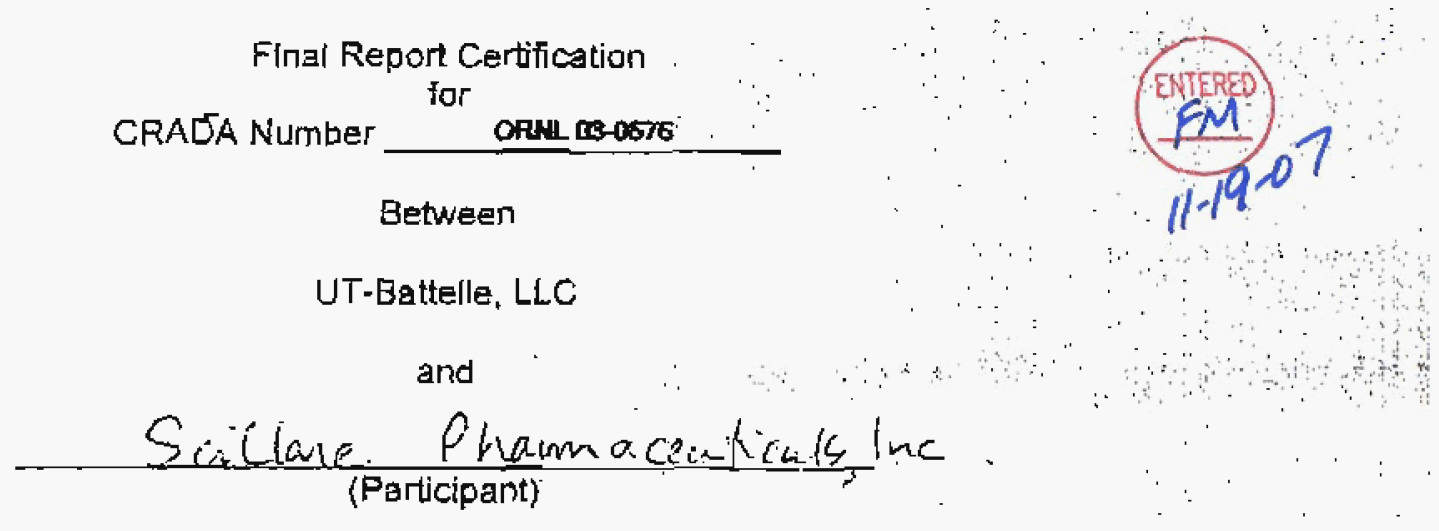

Instructiont:

Mark the appropllete staternent in $1 a$ ar $1 b$ below with an ' $X X$ * Refer to the articles In the CRADA terms and conditions governing the dontification and marking of Protacted CAADA infomation (PCl).

If no PCl is identifed, the report will be distributed without restiction. If PCI is identified, the report distribution will be lirnited in accordance with the CRADA terms and conditions governing release of data. In all ceses items 2 and 3 must be true. That is, the report cannor contain Proprietery Infomation and a disclosure must be filed prior to release of the report.

This cerification may either be made by using this form or may be made orr company letterhead if the Particlpant desires. A faxed copy of this completed form is accsptable

The following certification is made for the subject final repor:

1. (a) $\square$ The final report contains intormation that qualifies as "Protected CRADA Information" (PCI). The PCl legend is printed on the report cover, and the $P C l$ is clearly identified.

OR

(b) XIThe final report does not contain "Protected CRADA Infornation." The "Approved for Public Release" legend is printed an the seport cover.

2. The final report does not contain Proprietary Information.

3. By the signature below, the Participant has no objection to the public distribution of the final report due to patenlable information.

For the Participant.

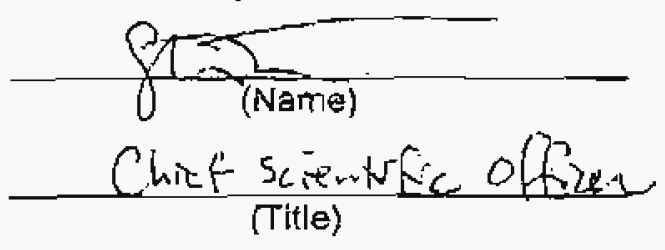

ORNL-251 (9RODT)

$$
\text { Na, } \frac{16,2007}{(\text { Date })}
$$




\title{
DEVELOPMENT OF AN ORAL FORMULATION OF SCV-07 FOR USE IN TUBERCULOSIS
}

ISTC Project No 2788

CRADA H ORNL 03-0676

Participant: SciClone Pharmaceuticals

\begin{abstract}
An evaluation of the immunomodulatory peptide SCV-07 was conducted as a possible therapeutic treatment for tuberculosis. This evaluation included mouse models, clinical trials and various forns of the drug such as liquid injection and development of an oral pill. It was found that SCV-07 sigrificantly increased the survival rate of arimals infected with lethal doses of Mycobacterium bovis. It enhanced the functional activity of macrophages in a dose-dependant fashion. The combination of SCV-07 with bacteriostatic drugs, such as izoniazid, was particularly effective. Phase JI clinical trials in a TB cliric demonstrated that the usage of the injection form of SCV-07 for lung TB treatment in combination with standard chemolherapy decreased the quantity of patients with positive sputum assays for Mycobacteria, promoled healing of cavities in lungs, stabilized parameters of cell immunity, and resulted in a significant improvement in the general condition of patients. Clinical trial results of the oral drug fom are still being evaluated.
\end{abstract}

OBJECTIVE

The general objective of this research project is the development and evaluation of the therapeutic potential of oral drug foms of the immunomodulatory peptide SCV-07 
for the treatment of tuberculosis (TB) since this form is the safest and most convenient drug formulation for clinical and ambulatory usage. Extended clinical trials of the injection form of SCV -07 for the treatment of non-pulmonary TB were also conducted.

Tuberculosis (TB) is a chronic infectious disease caused by the tubercle bacillus Mycobocterium - (including $M$. Luberculosis, $M$. bovis, $M$. africanum, and $M$. microti), which worldwide affects approximately $30 \%$ of the world's 5.7 billion people. More than 3 million people die of TB each year and TB kills more people today than does any other infectious agent. Some health experts view today's TB situation as similar to AIDS in the 1980 with TB quickly reaching pandemic proportions. This highlights why the development of new diagnostic methods, improvements in vaccine quality and a search for new therapeutic approaches for treatment of TB are of great importance.

Pulmonary TB is transmitted by the respiratory route. The likelihood of infection is determined by the severity of the infection in the infected person, the extent of exposure (duration, amount of coughing, degree of filtering or dilution of air, etc.), and the immune status of the infected person. If the infected person has a competent immune system, the growth and spread of bacteria is brought under control within two to ten weeks. However, even in these cases, viable bacilli remain in the macrophages in a domant state, and reactivation of the disease can occur at a later date.

Persons with weakened immune systems are particularly susceptible to TB, and infection with the human immunodeficiency virus (HIV) weakens the body's immune system, HV- positive persons have a much higher risk of developing active clinical TB. TB and HIV are synergistic infections - HIV infection speeds the progression of the TB disease and increases the activation rate of latent TB infection. TB appears to increase 
HIV replication in HIV infected individuals. The World Health Organization estimates that more than 5 million people world-wide are co-infected with HIV and TB, and approximately one-third of all HIV-related deaths world-wide are caused by TB.

Upon an initial infection by the TB microorganism, alveolar macrophages ingest the tuberculosis bacilli and transport them to regional lymph nodes. T lymphocytes become activated and release cytokines, interleukins and chemotactic factors. Peripheral monocytes enter the area and are transformed into aclivated macrophages by these secreted factors. Finally, the lymphocytes and macrophages wall off the sile of infection to form a granuloma. This process of cell-mediated imumunity requires approximately $3-9$ weeks. Due to the efficacy of the process about $90 \%$ of infected people never become clinically ill. However, defects in immune funclion greally increase the chance that clinical infection will occur. Moreover, clinical disease may recur if there is a reduction in nomal immune function.

The response of the human immune system to antigens, including infectious agents, is direcled by two different subsets of T-helper cells (referred to as type 1 and type 2, or Thl and Th2). The predominance of either the Thl or Th2 lymphocyle subset can result in different inflammatory pathways and disease outcomes. Research has shown that in mycobacterial infections, it is the CD4+ Th 1 cells and their associated type I cytokines (interleukin 2 [IL-2] and interferon $\gamma$ [IFNy]) which play a key role both in protective immunity and immunopathology. IFNy is considered to be the major macrophage activaling factor, which also plays a major role in suppressing a potentially diseasepromoting Th2 ingoune response. 
Multiple chemotherapeutic drugs for treatment of TB are available, including isoniazid, rifampin, pyrazinamide, and ethambutol. There are major drawbacks to these therapies however, including the prolonged length of treatment (>6 months) and a number of serious adverse reactions (including hepatitis, drug interactions, rumbness/tingling/pain in extremities, fatigue, flu-like symptoms, bleeding, rashes, stomach upset, and visual problems). In addition, and most importantly, the emergence of strains which are resistant to these agents have lead to dramatic outbreaks of multidrug-resistant disease (MDR-TB) - most notable in the HIV-infected populations in both the US and Europe.

SCV-07 (garmma-glutamyl-tryptophan) is one of a group of new immunomodulatory compounds that possess garnma-glutamyl or beta-aspartyl moieties, which were developed and patented both for composition and immunomodulatory use in Russia (patent Nos. 2091389 and 2120298 [11/28/95)]) and the USA (patent Nos. $5,744,452[4 / 28 / 98]$ and $5,916,878[1 / 29 / 99])$. As the most potent analog, SCV-07 has been shown to have a broad spectrum of immunostimulatory activities both in vitro and in vivo that can affect the differentiation of pluripotent stem cells to thymocytes and/or activation of thymocytes into T-cells with preferential activation of the Th1 lymphocyte subset. SCV-07 has been shown to increase Con-A-induced proliferation of thymocytes and lymphocytes, stimulate expression of Thy- l, 2 antigen on bone-marrow cells, and increase both production of IL-2 and expression of IL-2 receptors in response to Con-A in spleen lymphocytes. SCV-07 has also been shown to enlance the functional activity of peripheral blood leukocytes. A strong immunostimulatory effect of SCV-07 has been demonstrated in animals immunosuppressed by injection of 5-FU and in immunization 
models with sheep red blood cells. SCV-07 injections in mice immunized with protein antigen caused increased production of Th 1 cytokines such as IL-2 and IFN $\gamma$, but not of Thelper 2 cytokines. This implies that SCV-07 stimulates the immune response through preferential activation of the Thl lymphocyte subset.

$\mathrm{SCV}-07$ is thus appropriate as a therapeutic agent for infectious diseases associated with an inadequacy of Th1-mediated immunity, such as TB. SCV-07 is particularly well-suited for treatment of drug-resistant TB.

The primary focus of the project was the development of an SCV-07 drug formulation for oral delivery. This required: I) the development of immunological models in experimental animals for evaluation of SCV-07 activity after oral administration; 2) the development of an oral formulation of SCV-07; 3) an investigative study of SCV-07 anti-ruberculosis activity after oral administration in combination with tuberculostatic compounds; 4) evaluation of packaging and storage requirements; and 5) an evaluation of disease progression in laboratory models of lung tuberculosis.

The methods used in this project included classical imumunology and bacteriology techriques as well as molecular approaches:

- Lymphocyte er vivo proliferation studies using ${ }^{3} \mathrm{H}$-thymidine technique - Lymphocyte surface molecule expression studies

- Cytokine production in vivo and in cell cultures using ELISAs and biological testing on cytokine-dependent cell lines

- Th1 and Th2 lymphocyte subset analysis using cytokine production patterns and Ig subclasses titers

- PCR methods for detection of Mycobacterium tuberculosis

- A model of tuberculosis in mice, challenged with Mycobacterium bovis

Included in the project are expanded clinical trials of the SCV-07 injection formulation in a TB clinic where the influence of SCV-07 on the clinical and 
inmunological status of patients suffering from lung tuberculosis was evaluated (as well as the effect on extra-pulmonary localization). The optimal schedule of SCV-07 administration was also determined.

Additionally, a side experiment was also conducted to determine the effectiveness of SCV-07 as a radioprotectant, since preliminary evidence indicated that stimulation of the immune system could afford some radiological protection.

Male and female C57BL6/J mice were used for this study. For male and female sham and male SCV $-07, n=20 ; n=15$ for female SCV-07. Mice were injected intraperitoneally ( $1 \mathrm{mg} / \mathrm{kg} ; 0.105 \mathrm{ug} / \mathrm{ul}$ ) on the day of irradiation (7Gy; $\mathrm{Co}_{60}$ source) and every 3 days following irradiation. Sham mice received vehicle (PBS) alone. At 7 and 14-days after irradiation, 2 mice from each group were bled for hematology analysis. Mice were checked daily for deaths. At 17 days post-exposure, only a total of 4 SCV-07 mice survived. At this time point, all remaining mice were sacrificed and blood counts were analyzed.

WOC and WIC $=2$ different means of measuring WBC counts $\mathrm{RBC}=$ red blood cell number $\mathrm{HCT}=$ hematocrit

\begin{tabular}{|l|l|l|l|l|}
\hline 7 days post-irradiation & WOC & WIC & RBC & HCT \\
\hline Sham M & .036 & .120 & 7.72 & 38.1 \\
\hline Sham F & .032 & .183 & 7.66 & 36.9 \\
\hline SCV-07 M & .031 & .165 & 7.5 & 37.3 \\
\hline SCV-07 F & .093 & .142 & 8.34 & 41.1 \\
\hline
\end{tabular}




\begin{tabular}{|l|l|l|l|l|}
\hline 14 days post-juradiation & WOC & WIC & RBC & HCT \\
\hline Sham M & .037 & .132 & 4.09 & 18.3 \\
\hline Sham F & .032 & .081 & 3.35 & 15.8 \\
\hline SCV-07 M & .024 & .082 & 3.59 & 16.1 \\
\hline SCV-07 F & .026 & .096 & 3.17 & 14.3 \\
\hline
\end{tabular}

\begin{tabular}{|l|l|l|l|l|}
\hline $\begin{array}{l}\text { At sacrifice (17 days } \\
\text { post-imadiation) }\end{array}$ & WOC & WIC & RBC & HCT \\
\hline Sham M $(n=6)$ & .045 & .077 & 2.04 & 8.98 \\
\hline Sham F $(n=6)$ & .082 & .242 & 2.59 & 11.53 \\
\hline SCV-07M $(\mathrm{n}=3)$ & .141 & .347 & 2.88 & 13.1 \\
\hline SCV-07 F $(\mathrm{n}=1)$ & .029 & $\mathrm{nd}$ & 1.44 & 6.04 \\
\hline
\end{tabular}

\begin{tabular}{|l|l|l|l|l|}
\hline date & $\begin{array}{l}\text { Sham } \\
\text { male }\end{array}$ & Sham female & $\begin{array}{l}\text { SCV-07 } \\
\text { male }\end{array}$ & $\begin{array}{l}\text { SCV-07 } \\
\text { female }\end{array}$ \\
\hline $5 / 27-6 / 2$ & $\begin{array}{l}\text { No } \\
\text { deaths }\end{array}$ & No deaths & No deaths & No deaths \\
\hline $6 / 3$ & 2 & 1 & 5 & 3 \\
\hline $6 / 4$ & & & & 1 \\
\hline $6 / 5$ & 2 & 1 & 4 & 2 \\
\hline $6 / 6$ & 2 & 1 & 7 & 5 \\
\hline $6 / 7$ & 4 & 5 & & 1 \\
\hline $6 / 8$ & 1 & 1 & & \\
\hline $6 / 9$ & 3 & 5 & 1 & 2 \\
\hline TOTAL & $14 / 20$ & $14 / 15$ & $17 / 20$ & $14 / 20$ \\
\hline
\end{tabular}

The injection form of SCV-07 has passed all pre-clinical trials in Russia, and phase I and II clinical trials have been successively completed. All required documentation has been submitted to the Pharmacological and Pharmacopoeian Committees. Permission for clinical usage of SCV-07 as a medicine for treatment of 
secondary immunodeficiencies of different etiology was also granted. Additionally, SCV07 has been patented for both composition and immunomodulatory use in both Russia and the US.

Phase II clinical trials in the TB clinic demonstrated that usage of the injection form of SCV-07 for lung TB treatment, in combination with standard chemotherapy, decreased the number of patients with positive sputum assays for Mycobacterium, accelerated cavity healing, stabilized parameters of cell immunity, and resulted in significant improvement of the patients' general condition.

Success in the tasks outlined above led to a restricted clinical trial on volunteers suffering from lung tuberculosis which is currently being finalized. During this trial, in which patients will receive SCV-07 in combination with anti-TB chemotherapy, both clinical improvement and changes in lung condition are being evaluated, as well as the immunological status of the patierts.

The project is in conformity with the goals and lasks of ISTC and is aimed at development of an imsnunostimulatory drug for therapy of infectious diseases and secondary immunodeficiency. The results obtained from this project have extended cooperation between researchers of Russia and the USA, and is contributing significantly to the global prevention of the spread of tuberculosis.

\section{Conclusions:}

- Investigation of the specific biological activity of SCV-07 in the experimental model of lung TB in mice demonstrated the ability of SCV-07 to increase significantly the survival rate of animals infected with lethal doses of Mycobacterium bovis. It enhanced the functional activity of macrophages in a dose-dependant fashion. The combination of SCV-07 with bacteriostatic drugs, such as izoniazid, was particularly effective. Phase II clinical trials in a TB clinic 
demonstrated that the usage of the injection form of SCV-07 for lung TB treatment in combination with standard chemotherapy decreased the quantity of patients with positive sputum assays for Mycobacteria, promoted healing of cavities in lungs, stabilized parameters of cell immunity, and resulted in a significant improvement in the general condition of patients. Addition of SCV-07 to standard schemes of TB therapy also helped to decrease the period of usage of highly toxic chemotherapy drugs.

- Results (clinical improvement and changes in lung condition as assessed by chest $\mathrm{X}$-ray and bacteriological studies) from usage of the oral form of SCV-07 are still under investigation. The follow up period will be at least one year.

- No radioprotectant attributes were seen with SCV-07. 